\title{
Emfit Bed Sensor Activity Shows Strong Agreement with Wrist Actigraphy for the Assessment of Sleep in the Home Setting
}

\author{
Juan Piantino id \\ Madison Luther (iD) \\ Christina Reynolds ${ }^{2}$ \\ Miranda M Lim (D) ${ }^{2-4}$ \\ 'Department of Pediatrics, Division of \\ Child Neurology, Doernbecher \\ Children's Hospital, Oregon Health and \\ Science University, Portland, OR, USA; \\ ${ }^{2}$ Department of Neurology, Department \\ of Medicine, Division of Pulmonary and \\ Critical Care Medicine, Department of \\ Behavioral Neuroscience, Oregon Health \\ \& Science University, Portland, OR, USA; \\ ${ }^{3}$ Oregon Institute of Occupational Health \\ Sciences, Oregon Health \& Science \\ University, Portland, OR, USA; \\ ${ }^{4}$ Neurology Research Service and \\ National Center for Rehabilitative \\ Auditory Research, VA Portland Health \\ Care System, Portland, OR, USA
}

Purpose: Wrist-worn actigraphy via research-grade devices, a well-established approach to the assessment of rest-activity, is limited by poor compliance, battery life, and lack of direct evidence for time spent physically in the bed. A non-invasive bed sensor (Emfit) may provide advantages over actigraphy for long-term sleep assessment in the home. This study compared sleep-wake measurements between this sensor and a validated actigraph.

Patients and Methods: Thirty healthy subjects (6 to 54 years) underwent simultaneous monitoring with both devices for 14 days and filled out a daily sleep diary. Parameters included bed entry time, sleep start, sleep end, bed exit time, rest interval duration, and wake after sleep onset (WASO). The agreement between the two devices was measured using Bland-Altman plots and inter-class correlation coefficients (ICC). In addition, sensitivity, specificity, and accuracy were obtained from epoch-by-epoch comparisons of Emfit and actigraphy.

Results: Fifteen percent of the subjects reported that wearing the actigraph was a burden. None reported that using the bed sensor was a burden. The minimal detectable change between Emfit and actigraphy was 11 minutes for bed entry time, 14 minutes for sleep start, 14 minutes for sleep end, 10 minutes for bed exit time, 20 minutes for rest interval duration, and 110 minutes for WASO. Inter-class correlation coefficients revealed an excellent agreement for all sleep parameters (ICC $=0.99,95 \%$ CI 98-99) except for WASO (ICC $=0.46,95 \%$ CI $0.33-0.56$ ). Sensitivity, specificity, and accuracy were $0.62,0.93$, and 0.88 , respectively. Kappa correlation analysis revealed a moderate correlation between the two devices $(\kappa=0.55, \mathrm{p}<0.0001)$.

Conclusion: Emfit is an acceptable alternative to actigraphy for the estimation of bed entry time, sleep start, sleep end, bed exit time, and rest interval duration. However, WASO estimates are poorly correlated between the two devices. Emfit may offer methodological advantages in situations where actigraphy is challenging to implement.

Keywords: Actiwatch 2, Emfit, ambulatory, sleep-wake monitoring

\section{Introduction}

The assessment of sleep-wake patterns across days is an essential component of sleep medicine in clinical and research settings. The gold standard measurement of sleep is the polysomnogram (PSG). However, PSG is an invasive, expensive procedure limited to a single or few nights and unable to be routinely performed in specific populations (eg, infants, nursing home residents) or large cohort studies.

Actigraphy provides a low-cost, objective method to assess sleep used in both clinical and research settings. A growing number of studies have demonstrated the validity of actigraphy in distinguishing between sleep and wakefulness and
Correspondence: Juan Piantino

Email piantino@ohsu.edu 
providing reliable measures of sleep-wake organization and sleep quality, both in adults and children. ${ }^{1,2}$

However, although actigraphy devices are minimally invasive, studies using actigraphy have reported high rates of non-compliance as subjects do not wear the sensors or forget to press the event button to indicate bed entry and exit times. ${ }^{3-5}$ For that reason, actigraphy is often used in combination with daily sleep diaries completed by subjects or their caretakers. Studies have reported high levels of agreement for sleep start and end times between actigraphy and sleep diaries. ${ }^{6}$ However, in some instances, sleep diaries can be fraught with reporter bias or pose a significant burden to subjects. Discrepancies between actigraphy and sleep diaries are found in populations with memory problems, such as the elderly or those with cognitive impairment. ${ }^{5,7}$ Also, subjects with mood disorders can have a distorted perception of time, leading to over or under-reporting total sleep duration. ${ }^{8}$ Last, in specific populations such as children, there are significant differences between sleep estimates obtained by actigraphy and diaries filled by caregivers. ${ }^{9,10}$ Furthermore, most actigraphy devices have limited battery life and require recharging during studies longer than 1-4 weeks in duration. These limitations have restricted our understanding of the role of sleep both in health and disease.

For those reasons, a non-invasive, objective measurement of sleep that can be used for prolonged periods, with little burden to the subject or caregiver, is needed. Pressure sensors located under a standard bed mattress utilize highly sensitive plethysmography to assess presence and movement in the bed. They provide an objective assessment of time in and out of bed and are non-invasive. Some bed sensors have the added ability to detect and record other physiological signals, such as heart rate and respiratory rate. However, the reliability of these measurements has yet to be validated. Bed sensors do not depend on battery power and can theoretically be used as long as the subject has access to electricity in the home. More importantly, they do not require subjects to wear a device, increasing adherence to the protocol. These benefits over actigraphy make bed sensors a promising technology in the long-term assessment of sleep in the home. Despite these advantages, these novel devices still require validation against accepted methodologies (ie, actigraphy).

In this study, we aimed to compare different sleep elements obtained with both actigraphy and a bed sensor in the home setting in a cohort of healthy individuals.

\section{Patients and Methods}

\section{Study Design and Participants}

This is a cross-sectional study. Subjects were enrolled between May and August 2020. Written informed consent for study inclusion was obtained from a parent or legal guardian for all minors (5-17 years). Written informed consent was collected from adults aged 18 and older. The study was approved by the Oregon Health \& Science University Institutional Review Board, and was conducted in accordance with the Declaration of Helsinki. Thirty subjects (17 women) were recruited from the community via flyers and direct contact. Subjects were eligible to participate in the study if a) they were between the ages of 5 to 65 years; $b$ ) they were able to provide consent; c) they slept the majority of nights in the same bed. Subjects were excluded if: a) they could not follow the study protocol (ie, complete the sleep diary and exit survey); b) they did not sleep most nights in the same bed. Subjects were not excluded based on the presence of a sleep disturbance or based on whether they slept alone or co-slept.

\section{Variables}

\section{Clinical and Demographic Variables}

These included age, weight, height, race, sleep habits (whether the subject sleeps alone or co-sleeps), and self-reported history of sleep problems (ie, insomnia, sleep apnea, sleepwalking, sleep paralysis, sleep terrors, restless legs, narcolepsy).

\section{Assessment of Sleep}

Sleep data was captured for 14 days. Based on previous work on the agreement between actigraphy and sleep diaries, ${ }^{5}$ subjects were included in the final analysis if they contributed at least three valid nights. A night's data was considered invalid if: a) the sleep diary was incomplete, b) the actigraphy data were missing (ie, the subject did not wear the watch for the entirety of the night, or the watch malfunctioned), and c) the bed sensor data were missing (ie, the bed sensor malfunctioned, or subjects did not sleep in their bed).

\section{Actigraphy}

We used the Philips Actiwatch 2 (Philips Respironics, Bend, OR). The Actiwatch 2 device provides actigraphy data and ambient light levels derived from raw accelerometer and photometer values. Participants were instructed to wear the watch on their non-dominant wrist while continuing their normal activities and sleep in their home environment. The actigraphy sensor uses acceleration data sampled at $32 \mathrm{~Hz}$ to calculate an activity count, which equals the sum of peak activity values for 
each second of data monitored over a 1-minute epoch. A score $=\mathrm{E}_{(-2)} *(1 / 25)+\mathrm{E}_{(-1)} *(1 / 5)+\mathrm{E}_{0}+\mathrm{E}_{(+1)} *$ $(1 / 5)+E_{(+2)} *(1 / 25)$, with $E_{0}$ being activity counts for the epoch being scored, and $E_{(n)}$ being the activity counts for the epochs $n$ seconds preceding or following $E_{0}$. Raw data were recorded and binned each minute for 14 continuous days. For the epoch-by-epoch analysis, a subject was considered "awake" if an epoch contained an activity count above 20 (low setting).

\section{Bed Sensor}

The Emfit movement monitor (Emfit Corp., Kuopio, Finland) is a flexible movement sensor ( $32 \mathrm{~cm} \times 62 \mathrm{~cm} \times 0.4 \mathrm{~cm})$ placed under the mattress at the thoracic level. If a subject co-sleeps, the sensor is placed under their side of the bed. Emfit has been used in the clinical setting to detect movements in sleeping subjects. ${ }^{11}$ The sensor consists of thin elastic, lightweight polymer layers separated by air voids and coated with electrically conductive, permanently polarized layers. ${ }^{12}$ Pressure changes acting on the film generate a charge on its electrically conductive surface measured as a voltage signal. ${ }^{13}$ The raw signal is acquired continuously throughout the day at $200 \mathrm{~Hz}$. Each device is equipped with a Subscriber Identification Module (SIM) card, which transmits data in real-time to a secure, encrypted server. Subjects are not required to own a cell phone or have WiFi installed at their homes for the device to work. The data is subsequently filtered into two different frequency bands, as documented in previous work. ${ }^{14}$ This continuous recording allows the bed sensor to detect bed entry and exit times (see definitions below) without the need for a sleep diary. As with actigraphy, the raw actigraphy data were recorded and binned each minute for the duration of the study. These data were used to manually quantify large body movements (entry into bed, exit out of bed) and determine whether a subject was asleep or awake for every 1-minute epoch (see below). The low-frequency band (LF, 0.3-10 Hz) was used to visualize respiratory movements, whereas the high-frequency band (HF, 6-16 Hz) was used to visualize heart rate and respiratory-related spikes. ${ }^{15}$ Heart rate and respiratory rate were not used in the current analysis and thus are not reported here.

\section{Sleep Diary}

During the 14-day study period, participants were instructed to fill out a daily sleep diary, in which they indicated bed entry time, time asleep, time awake, and bed exit time. The sleep diary was delivered via text message to the subject's cell phone at the same time each day (8:00 am) via a third-party application (Twilio, Inc. San Francisco, CA). Automated reminder text messages were sent every 2 hours for a total of 3 tries, or until the subject responded. This method allowed the study coordinator to monitor adherence to the protocol daily.

A detailed protocol outlining the process for visual scoring of sleep periods based on previous literature ${ }^{16}$ can be found in Supplementary Table 1. The data from the actigraphy watch and bed sensor was scored by a single scorer and was categorized as follows:

- Bed entry and exit times. Actigraphy: because of potential limitations with the automated marking of rest intervals, Actiwatch user manual (Philips Actiware 6.0) instructions advise researchers to inspect the data and manually set the rest intervals. ${ }^{17}$ Based on these recommendations and previous work validating manual marking of these intervals, ${ }^{16}$ bed entry and exit times were identified manually according to a standardized protocol, which, in previous studies, has demonstrated high correlations between scorers (intraclass correlation $=0.9$ ). ${ }^{16}$ For actigraphy, bed entry time was defined using the following information: a) pronounced drop in activity level (less than one activity count per 15 seconds); b) decreased ambient light detected by the actigraphy watch; c) subject's sleep diary. Bed exit time was defined using the following information: a) periods of increased mobility (more than one activity count per 15 seconds) after sleep end; b) increased ambient light; c) subject's sleep diary. Bed sensor: bed entry and exit times were defined as the first and last movements detected by the bed sensor, respectively. - Sleep start and end. Based on previous work, ${ }^{5}$ sleep start and end were defined as follows for both devices. Sleep start: the first immobile period of at least 10 minutes after bedtime with no more than one epoch of movement. Sleep end: the last period of at least 10 minutes of immobility before get up time that had no more than one epoch of movement.

- Rest interval duration (nocturnal sleep period) was calculated as the difference between sleep start and sleep end.

- Wake After Sleep Onset (WASO), defined for both devices as the number of 1-minute epochs spent awake between sleep start and sleep end.

\section{Questionnaires}

At the end of the 14-day recording, all subjects received an 8 -item exit questionnaire via text message to assess their 
experience with both devices. Each answer consisted of a 5-point Likert scale (range: 1=" strongly disagree," 2="“ disagree," $3=$ "“ neutral," $4=$ "agree," $5=$ "“ strongly agree”).

\section{Statistical Analysis}

Statistical analysis was performed using Stata/MP 15 (StataCorp, College Station, TX). Descriptive statistics were used to summarize the cohort's characteristics. We evaluated the level of agreement between the bed sensor and actigraphy with three independent methods. First, we used the method described by Bland and Altman ${ }^{18}$ to calculate the mean of the difference between the two devices (bed sensor minus actigraphy). The normality of the difference between the two devices was tested with Shapiro and Wilk's test. The difference in sleep start was normally distributed. Bed entry time, sleep end, bed exit time, rest interval duration, and WASO were not normally distributed. Still, the deviations were not substantial (Q-Q plots can be found in Supplementary Figure 1). When analyzing Bland-Altman plots, we report the 95\% limits of agreement, the minimal detectable change, and the minimal clinically important change as recommended by Haghayegh et al. ${ }^{19}$ The $95 \%$ limits of agreement between the two devices are defined as the mean difference $\pm 1.96 \mathrm{SD}$ of the differences. The minimal detectable change is defined as the smallest amount of change in the score detected by a method, independent of measurement error. It is calculated as one-half of the difference between the upper and lower limit of agreement of the BlandAltman plot. ${ }^{20}$ The minimal clinically important change is defined as the smallest change in the outcome measure considered important by the investigators. A priori, and based on previous sleep validation studies, ${ }^{10}$ we established a minimal clinically important change of $30 \mathrm{~min}-$ utes. Second, we calculated the inter-class correlation coefficients (ICC) ${ }^{21}$ with a two-way random effects, absolute agreement model (ie, each target was rated by the same set of raters, in this case, the bed sensor and actigraphy, and the measurement of interest is the absolute agreement between the two). We report ICCs with their respective $95 \%$ confidence intervals. Of note, when $p$-values for ICC between two measurements are calculated, they test the null hypothesis that such measurements were generated at random $(\mathrm{ICC}=0)$. For that reason, it is possible to have a low correlation between the two measurements and still have a $p$-value less than the conventionally accepted 0.05 . Though more stringent, a widely accepted method is to report ICC and their respective $95 \%$ confidence intervals. In this approach, ICC is categorized as poor (ICC values less than 0.5 ); moderate (ICC values between 0.5 and 0.75 ); good (ICC values between 0.75 and 0.9); and excellent (ICC values greater than 0.9) based on the lowest limit of the $95 \%$ confidence interval. ${ }^{22}$ Third, we assessed the agreement between the two devices for each 1-minute epoch using the method used by Pollak et $\mathrm{al}^{23}$ and replicated in subsequent actigraphy validation studies. ${ }^{24}$ Briefly, each time the bed sensor's data agreed with actigraphy data, an epoch was classified as "true sleep" or "true awake." When the bed sensor and actigraphy disagreed, epochs were classified as "false sleep" or "false awake." The bed sensor's sensitivity (the ability to detect sleep when actigraphy scores it as sleep) is then calculated as the number of true sleep epochs divided by the number of actigraphy sleep epochs. Specificity (the ability to detect wake via the bed sensor when actigraphy scores it as wake) is calculated as the number of true awake epochs divided by the number of actigraphy awake epochs. Accuracy (the ability to detect both sleep and wake compared to actigraphy) is calculated as the number of true sleep + true awake epochs divided by the sum of all actigraphy-scored epochs. Last, we calculated the Cohen's kappa coefficient between the two devices, with the interpretation suggested by Landis and Koch. ${ }^{25}$

Although not part of our primary aims, we explored differences in the estimation of all sleep parameters between the two devices in subjects with insomnia versus subjects with no insomnia. We performed a two-tailed $t$-test with insomnia status as the independent variable and the difference between the two devices for each sleep parameter as the dependent variable. We used Bonferroni correction to adjust for multiple comparisons.

\section{Results}

\section{Cohort Characteristics}

A total of 30 subjects (including 17 women), aged 6 to 54 years (median 25, interquartile-range 16 to 43 ), participated in the study. Ten subjects (33\%) were younger than 18 years (Table 1). Thirteen subjects (43.3\%) were co-sleepers. None of the subjects younger than 18 years were co-sleepers. All the study participants fulfilled the criterion of three valid nights. On average, subjects had 13.5 nights of bed sensor data available for analysis (range 10-14) and 11.5 nights of actigraphy data available for analysis (range 6 to 14). However, only nights with both bed sensor and actigraphy 
Table I Cohort Characteristics

\begin{tabular}{|c|c|}
\hline Characteristics & Cohort $(n=30)$ \\
\hline Age (yrs), median (IQR) & $25(16-43)$ \\
\hline Age <18 years, $n(\%)$ & $10(33)$ \\
\hline Female sex, $\mathrm{n}(\%)$ & $17(56.6)$ \\
\hline Height (in), median (IQR) & $65.5(62-70)$ \\
\hline Weight (Ibs), median (IQR) & $146.5(125-168)$ \\
\hline \multicolumn{2}{|l|}{ Race, n (\%) } \\
\hline Asian & $4(13.3)$ \\
\hline White & $25(83.3)$ \\
\hline Multiracial & I (3.3) \\
\hline \multicolumn{2}{|l|}{ Sleep habits, n (\%) } \\
\hline Sleeps alone & $17(56.6)$ \\
\hline Co-sleeps & $13(43.3)$ \\
\hline \multicolumn{2}{|l|}{ Sleeping problems ${ }^{\mathrm{a}}, \mathrm{n}(\%)$} \\
\hline Insomnia & $2(6.6)$ \\
\hline Sleep apnea & I (3.3) \\
\hline Night terrors & I (3.3) \\
\hline Nights recorded per patient, mean (range) & II (6-14) \\
\hline
\end{tabular}

Notes: ${ }^{a}$ No subjects reported sleepwalking, sleep paralysis, restless leg syndrome, or narcolepsy.

Abbreviation: IQR, inter quartile range.

data were included in the final analysis. This led to a final dataset of 332 nights, with a final average of 11 (range 6-14) nights per participant (Figure 1). Nights were excluded for the following reasons: a) missing actigraphy data (the subject did not wear the watch, $n=66$; the watch malfunctioned, $n=7), b$ ) missing bed sensor data (ie, sensor became unplugged or lost connection to the internet $(\mathrm{n}=10)$, or subjects did not sleep in their bed, $n=4), c)$ missing both bed sensor and actigraphy data $(n=1)$. Specific examples of excluded nights showing actigraphy, bed sensor, and sleep diary data are presented in Supplementary Figure 2. Of note, subjects completed the sleep diaries for all 420 nights; therefore, no nights were excluded based on missing sleep diary data.

\section{Acceptability, Feasibility, and Adherence}

Of the 30 subjects included in the cohort, 27 (90\%) completed the exit questionnaire. Four subjects $(15 \%)$ reported that wearing the actigraph was a burden. None reported that using the bed sensor was a burden. Five subjects $(18.5 \%)$ reported finding it difficult to wear the watch over an entire month. One subject (3.7\%) reported finding it difficult to use the bed sensor over a whole month. If sleep monitoring were to be done over a month, 22 subjects $(81.4 \%)$ reported the bed sensor as the only method they would prefer, whereas 5 subjects $(18.5 \%)$ chose both the bed sensor and actigraphy.

\section{Sleep Parameters \\ Bed Entry Time and Sleep Start}

A summary of the differences in sleep estimates between the bed sensor and actigraphy can be found in Table 2. On average, estimates of bed entry time and sleep start by the bed sensor were 1 minute earlier (95\% CI -2 to -1 minutes) and 1 minute later (95\% CI 0 to 2 minutes) than those estimated by actigraphy. Bland-Altman plots revealed a minimal detectable change of 11 minutes for bed entry time and 14 minutes for sleep start. Interclass correlation coefficients revealed an excellent correlation between the two devices for both estimates (ICC 0.99 [95\% CI 0.99 to 0.99]). Examples of raw activity data showing agreement between the two devices can be found in Figure 2.

\section{Sleep End and Bed Exit Time}

On average, estimates of sleep end and bed exit time by the bed sensor were 0 minutes ( $95 \% \mathrm{CI}-1$ to 1 minute) and 1 minute (95\% CI 1 to 2 minutes) later than those estimated by actigraphy. Bland-Altman plots revealed a minimal detectable change of 14 minutes for sleep end and 10 minutes for bed exit time. Interclass correlation coefficients revealed an excellent correlation between the two devices for both estimates (ICC 0.9 [95\% CI 0.99 to 0.99$])$.

\section{Rest Interval Duration and WASO}

On average, estimates of rest interval duration by the bed sensor were 1 minute shorter than those estimated by actigraphy (95\% CI -2 to 0 minutes). Bland-Altman plots revealed a minimal detectable change of 20 minutes. Interclass correlation coefficient revealed an excellent correlation between the two devices for rest interval duration estimates (ICC 0.98 [95\% CI 0.98 to 0.98]). On average, WASO estimates by the bed sensor were 3 minutes longer than those estimated by actigraphy (95\% CI -2 to 9 minutes). Bland-PAltman plots revealed a minimal detectable change of 110 minutes. Interclass correlation coefficient revealed a poor correlation between the two devices for WASO estimates (ICC 0.46 [95\% CI 0.33 to 0.56$]$ ). Of note, in individuals with insomnia $(n=2)$, the mean differences in estimates of all sleep parameters were no different than those with no insomnia after adjusting for multiple comparisons (data not shown). 
Met eligibility and enrolled $(n=30)$

1. Age: $5-65$ years

2. Able to provide consent*

3. Sleeps the majority of nights in the same bed

\begin{tabular}{|l|l|}
\hline & \begin{tabular}{|l|} 
Excluded nights due to missing sleep diary \\
data $(n=0)$
\end{tabular} \\
& $\begin{array}{l}\text { Excluded nights due to missing actigraphy } \\
\text { data }(n=73) \\
1 . \quad \text { Subject did not wear the watch }(n=66) \\
2 . \quad \text { Watch malfunctioned }(n=7)\end{array}$ \\
\hline $\begin{array}{l}\text { Final sample size: } \\
\text { 1. Total subjects }(n=30) \\
\text { 2. Total nights }(n=322)\end{array}$ & $\begin{array}{l}\text { Excluded nights due to missing mattress } \\
\text { sensor data ( } n=14)\end{array}$ \\
\hline
\end{tabular}

Figure I Enrollment flow chart. *Consent obtained from a parent or legal guardian for all minors (5-17 years).

To further investigate the effects of age on the agreement between the two devices, subjects were stratified between those younger than 18 years $(n=10)$ and those 18 years or older $(n=20)$. Interclass correlation coefficients revealed an excellent correlation between the two devices for bed entry, sleep start, sleep end, bed exit, and rest interval duration (Supplementary Table 2). There was a poor correlation between the two devices for WASO in subjects younger than 18 years (ICC 0.32 [95\% CI -0.01 to 0.55 ), but a moderate correlation for subjects older than 18 years ( $\mathrm{ICC}=0.68$ ( 0.59 to 0.75$)$. Interclass correlation coefficients also revealed an excellent correlation between the two devices for bed entry, sleep start, sleep end, bed exit, and rest interval duration when subjects were stratified by those who sleep alone versus co-sleepers (Supplementary Table 3). There was a poor correlation for WASO among those subjects who sleep alone (ICC 0.36 [0.14 to 0.53$]$ ), but a moderate correlation among cosleepers (ICC 0.74 [0.65 to 0.81$]$ ).

\section{Sensitivity, Specificity, and Accuracy}

A comparison of all epoch classifications by actigraphy and the bed sensor is presented in Table 3. A total of 188,576 one-minute epochs were analyzed. The bed sensor had a sensitivity of 0.62 , specificity of 0.93 , and accuracy of 0.88. Cohen's kappa coefficient revealed a moderate correlation between the two devices $(\kappa=0.55, \mathrm{p}<0.0001)$.

\section{Discussion}

This cross-sectional study found an excellent correlation between a bed sensor and an actigraphy watch for bed entry time, sleep start, sleep end, bed exit time, and rest interval duration. There was a poor correlation between the two WASO estimates among subjects younger than 18 years but a moderate correlation among subjects older than 18 years. Compared with actigraphy, the bed sensor showed high specificity and accuracy but lower sensitivity. Fifteen percent of our subjects found wearing the actigraph burdensome, whereas none found using the bed sensor burdensome. 
Table 2 Correlation of Sleep Parameters Recorded by Emfit Bed Sensor and Actigraphy

\begin{tabular}{|c|c|c|c|c|c|c|}
\hline & \multicolumn{2}{|c|}{$\begin{array}{l}\text { Measurement Type (h: } \\
\text { min), Mean (SD) }\end{array}$} & \multirow{2}{*}{$\begin{array}{c}\text { Difference } \\
\text { (min), Mean } \\
(95 \% \mathrm{Cl})^{\mathrm{a}}\end{array}$} & \multirow{2}{*}{$\begin{array}{l}\text { Bland-Altman } \\
\text { 95\% Limits of } \\
\text { Agreement } \\
\text { (h:min) }\end{array}$} & \multirow{2}{*}{$\begin{array}{c}\text { Bland-Altman } \\
\text { Minimal } \\
\text { Detecteble } \\
\text { Change (min) }\end{array}$} & \multirow{2}{*}{$\begin{array}{l}\text { Inter-Caclass } \\
\text { Correlation } \\
\text { Coefficient ( } 95 \% \\
\text { Cl) }\end{array}$} \\
\hline & Emfit & Actigraphy & & & & \\
\hline Bed entry time & $22: 31(1: 30)$ & $22: 32(1: 29)$ & $-1(-2$ to -1$)$ & -12 to 10 & 11 & 0.99 (0.99 to 0.99$)$ \\
\hline Sleep start & $23: 21(1: 35)$ & $23: 20(1: 35)$ & I (0 to 2$)$ & -13 to 15 & 14 & 0.99 (0.99 to 0.99 ) \\
\hline Sleep end & $7: 23(1: 35)$ & $7: 23(1: 35)$ & $0(-I$ to $I)$ & -14 to 14 & 14 & 0.99 (0.99 to 0.99$)$ \\
\hline Bed exit time & $8: 10(1: 46)$ & 8:08 (1:46) & I (I to 2$)$ & -9 to II & 10 & 0.99 (0.99 to 0.99 ) \\
\hline Rest interval duration & 8:0I (I:27) & $8: 03(1: 27)$ & $-1(-2$ to 0$)$ & -21 to 19 & 20 & 0.99 (0.98 to 0.99$)$ \\
\hline WASO $^{c}$ & $56.5(60.4)$ & $53.1(26.8)$ & $3(-2$ to 9$)$ & -107 to 113 & 110 & $0.46(0.33$ to 0.56$)$ \\
\hline
\end{tabular}

Notes: ${ }^{a}$ Emfit minus actigraphy. ${ }^{\mathrm{b}}$ Minimum Detectable Change $=$ (upper limit of agreement - lower limit of agreement) $/ 2$.

Abbreviation: 'WASO, Wake after sleep onset (min).

In our study, we used various methods to determine the degree of agreement between the two devices. The method proposed by Bland and Altman offers the advantage of establishing limits of agreement with a $5 \%$ probability of error and has been adopted by various sleep validation studies. ${ }^{10,18,26}$ In a study of 50 healthy children, Werner et al used Bland-Altman plots to determine the degree of agreement between actigraphy and a sleep diary. ${ }^{10}$ A priori, the authors established a 30-minute time interval as the minimal clinically important change. In other words, a difference of 30 minutes or more between the two methods in the estimation of sleep parameters was considered of clinical significance. ${ }^{19,20}$ In our study, the differences between the two devices fell within 30minutes for all the parameters examined, except for WASO. Despite our findings, whether a 30-minute cutoff is acceptable for the interchangeable use of different sleep assessment modalities remains a question that needs to be answered from the clinical perspective. In addition to the Bland-Altman analysis, we measured the correlation between the two devices using ICC. Our analysis shows a moderate to excellent ICC for all sleep parameters except for WASO estimates among subjects younger than 18 years. The ICC for WASO is also higher among cosleepers, probably indicating that younger subjects are more likely to sleep alone. WASO is a measurement of

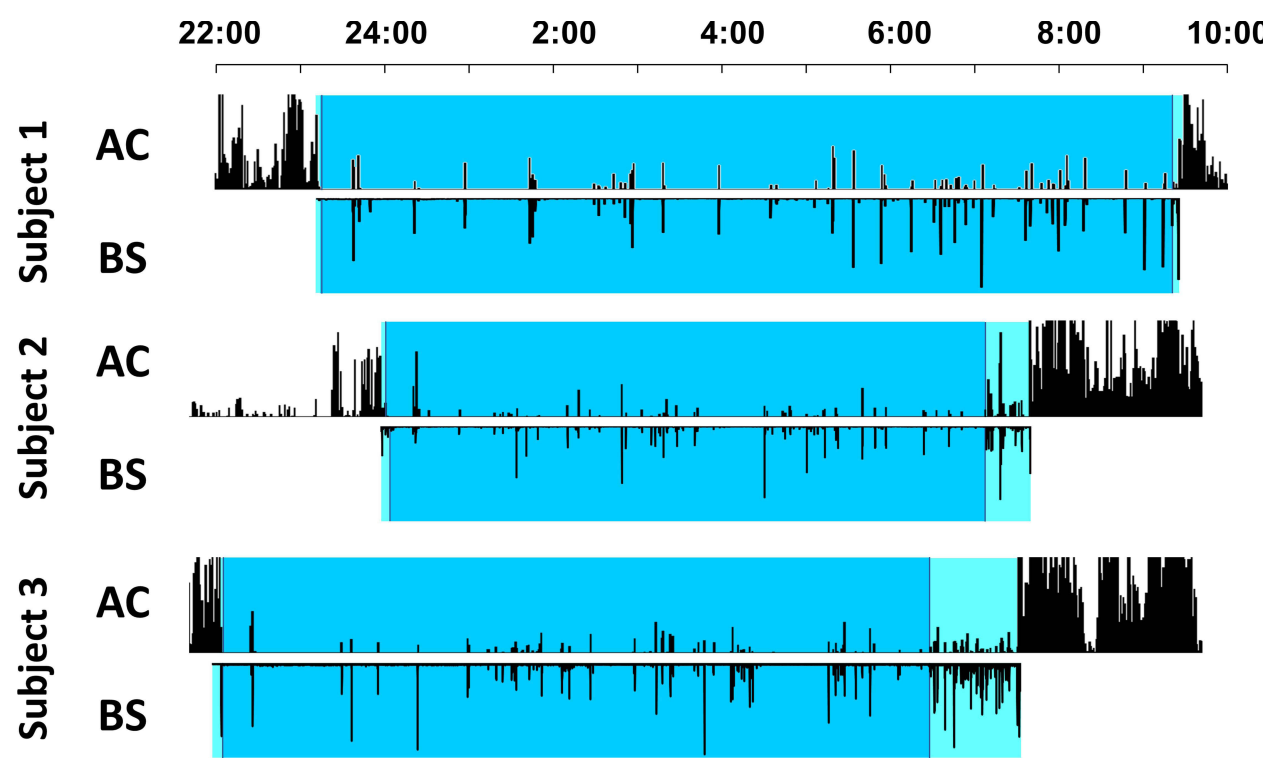

Figure 2 Synchronized movement data from actigraphy and bed sensor. Activity data for three different subjects, obtained with actigraphy (AC) and the bed sensor (BS). Light blue indicates the period between bed entry time and bed exit time. Dark blue indicates the period between sleep start and sleep end. Note how activity spikes (black) correlate between the two devices for all three subjects. Subjects 2 and 3 spend time between waking up (increase in activity noted by the two devices) and bed exit (noted by continuous activity on actigraphy and end of activity detected by the bed sensor). 
Table 3 Statistical Measures of Epoch-by-Epoch Comparisons Between Actigraphy and Emfit ${ }^{\mathrm{a}}$

\begin{tabular}{|l|l|l|l|l|}
\hline \multicolumn{2}{|l|}{} & \multicolumn{2}{|l|}{ Actigraphy } & \multirow{2}{*}{ Total } \\
\cline { 3 - 4 } \multicolumn{2}{|l|}{} & Sleep & Wake & \\
\hline \multirow{2}{*}{ Emfit } & Sleep & 149,657 & 10,681 & 160,338 \\
& Wake & 10,335 & 17,903 & 28,238 \\
& Total & 159,992 & 28,548 & 188,576 \\
\hline \multirow{2}{*}{ Sensitivity =0.62 } & Specificity $=0.93$ & Accuracy $=0.88$ & Kappa $=0.55^{\text {b }}$ \\
\hline
\end{tabular}

Notes: ${ }^{\mathrm{a}}$ Table entries are I-minute epochs. ${ }^{\mathrm{b}} \mathrm{p}$-value $<0.000 \mathrm{I}$.

sleep disruption commonly used in clinical and research settings. These discrepancies should be considered when selecting one device over another to monitor sleep patterns, particularly among younger individuals.

This study found the bed sensor to be excellent in detecting wake with a specificity of 93.5. The accuracy was also high at 88.8 . However, the sensitivity (ie, the bed sensor's ability to detect sleep) was lower at 0.62 . The bed sensor, which is placed under the mattress, may detect movements not detected by the actigraphy watch, particularly the lower limbs. Comparative studies between the bed sensor, actigraphy, and PSG are needed to clarify these observations.

Our study has several strengths. First, all measurements were taken at home, with minimally invasive methods, which produced negligible distortion in sleep patterns. Second, our cohort consisted of subjects with a wide age range (the youngest subjects were 6 years old). This age range was chosen in order to provide agreement data for both children and adults. Third, although both Phillips Respironics Inc. and Emfit Inc. provide sleep detection algorithms, we manually reviewed and scored all the sleep recordings. We make no claims as to the accuracy of Emfit's commercially available, automated algorithm. Several studies have demonstrated acceptable agreement rates between automatic scoring of actigraphy and PSG. ${ }^{27-30}$ However, in a large study of individuals with insomnia, Hauri and Wisbey found discrepancies of up to 49 minutes of rest interval duration between actigraphy scored using automatic algorithms and PSG. ${ }^{31}$ More importantly, in a study using Actiwatch 2, Chow et al demonstrated that automated algorithms could not accurately detect rest intervals compared to manual scoring. ${ }^{16}$ Although our study shows a high correlation between manually scored bed sensor and actigraphy data, this is a lengthy process that requires expertise and may not be feasible in large studies. Further research is needed to establish the correlation between different automated methods and direct human observation.
Our findings are particularly useful for those practitioners who need to assess sleep parameters in situations where traditional PSG, sleep diaries, or actigraphy may be challenging to implement. These situations include documentation of sleep patterns in the home setting, over prolonged periods, or in specific populations. For instance, persons with lower cognitive functioning, psychopathology, or other neurological problems often have difficulties filling out sleep diaries. ${ }^{7,32}$ Our data shows agreement between the bed sensor data alone versus hand-curated diary plus actigraphy data. These findings are of relevance to situations in which sleep diaries are difficult to obtain. In our study, more subjects in our cohort found actigraphy to be burdensome when compared to the bed sensor. In addition, the majority of subjects chose the bed sensor as their only preferred method for sleep monitoring over a one-month period. To date, most devices designed to offer an alternative to wrist-worn actigraphy remain at the prototype stage or have been insufficiently validated. ${ }^{33}$ Our data suggest that bed sensors might provide a reliable, feasible, and less burdensome alternative to actigraphy.

Our study has several limitations. First, actigraphy is not considered the gold standard for the assessment of sleep. Furthermore, actigraphy does not assess electroencephalographic manifestations of sleep, including the distinction between rapid eye movement (REM) versus non-REM sleep. Despite these limitations, actigraphy offers clear advantages over the gold standard (polysomnography), particularly in the ambulatory setting, and is recognized by the American Academy of Sleep Medicine as a valid method in determining sleep patterns. ${ }^{1,2}$ Our main goal was to compare two technologies with similar applications in the home setting and advantages across specific populations or situations. In their study, Choi et al compared sleep-wake measurements obtained with "bed actigraphy" (measured by load-sensing cells supporting the bed) and PSG. ${ }^{34}$ The mean epoch-byepoch agreement between the two devices was $95.2 \%$. A similar comparison between the bed sensor used in our study and PSG would be desirable. However, such a comparison alone would not address our scientific question because PSG is not routinely used in longitudinal, population-based, prospective studies of sleep. In addition to documenting agreement among different sleep measuring techniques, research also needs to focus on understanding the sources of discrepancies and promote their complementary use. Second, heart and respiratory rates (HR/RR), provided by the bed sensor, were not included in the analysis. 
The bed sensor provides HR/RR data, but the actigraphy watches do not. Our goal was to validate both devices using similar rating methods. While HR/RR do change with sleep, there are no established criteria for the definitive scoring of sleep based on changes in these vital signs, and differences in HR/RR between non-REM and REM sleep would further complicate the distinction of REM from wake on the basis of HR/RR alone. Also, to our knowledge, there are no published studies supporting the validity and reliability of HR/RR measurements by the bed sensors during different stages of sleep. Third, our cohort mainly consisted of healthy individuals. Agreement between the two devices may be lower in certain subjects (eg, patients with sleep disorders or depression) who often lay in bed motionless without sleeping. ${ }^{35}$ We did not find agreement differences when comparing subjects with insomnia versus those without insomnia. However, this was an exploratory analysis, and the study was not powered to detect small discrepancies. Last, we did not inquire whether subjects slept with a pet, which could have influenced sleep sensor data.

The bed sensor may be particularly useful in situations where adherence to actigraphy monitoring devices is low. However, despite the bed sensor's advantages, actigraphy offers certain benefits. For instance, the bed sensor does not provide daytime activity levels, which can be used to deduce lifestyle, and to some extent, circadian rhythm. In addition, actigraphy may still be preferred in subjects who do not sleep in the same bed consistently. When designing a sleep-related study, investigators are encouraged to take into account the characteristics of the study population and to utilize various measurements of sleep in order to get more accurate information regarding sleep patterns.

\section{Acknowledgments}

The authors would like to express their sincere appreciation and gratitude for the participation of all participants.

\section{Author Contributions}

1) Conception and design of the study: JAP, MML

2) Acquisition and analysis of data: JAP, ML, CLR

3) Drafting a significant portion of the manuscript or figures (ie, a substantial contribution beyond copy editing and approval of the final draft, which is expected of all authors): JAP, MML.

\section{Funding}

National Institutes of Health, National Heart, Lung and Blood Institute: K23HL150217, to J.A.P. VA CSRD Merit
Review Award \#I01 CX002022 and National Hartford Center of Gerontological Nursing Excellence (NHCGNE) \#1121 to M.M.L. NIH P30 AG066518-02 to C.L.R. and M.M.L.

\section{Disclosure}

This material is the result of work supported with resources at the VA Portland Health Care System. Interpretations and conclusions are those of the authors and do not represent the views of the US Department of Veterans Affairs or the United States Government.

The authors report no conflicts of interest in this work.

\section{References}

1. Morgenthaler T, Alessi C, Friedman L, et al. Practice parameters for the use of actigraphy in the assessment of sleep and sleep disorders: an update for 2007. Sleep. 2007;30(4):519-529. doi:10.1093/sleep/ 30.4.519

2. Smith MT, McCrae CS, Cheung J, et al. Use of actigraphy for the evaluation of sleep disorders and circadian rhythm sleep-wake disorders: an American academy of sleep medicine clinical practice guideline. J Clin Sleep Med. 2018;14(7):1231-1237. doi:10.5664/ jesm. 7230

3. Hoffman NL, O'Connor PJ, Schmidt MD, Lynall RC, Schmidt JD. Relationships between post-concussion sleep and symptom recovery: a Preliminary Study. $J$ Neurotrauma. 2020;37(8):1029-1036. doi:10.1089/neu.2019.6761

4. Zibrandtsen IC, Hernandez C, Ibsen JD, Kjaer TW. Event marker compliance in actigraphy. $J$ Sleep Res. 2020;29(1):e12933. doi:10.1111/jsr.12933

5. Van Den Berg JF, Van Rooij FJ, Vos H, et al. Disagreement between subjective and actigraphic measures of sleep duration in a population-based study of elderly persons. J Sleep Res. 2008;17 (3):295-302. doi:10.1111/j.1365-2869.2008.00638.x

6. Thurman SM, Wasylyshyn N, Roy H, et al. Individual differences in compliance and agreement for sleep logs and wrist actigraphy: a longitudinal study of naturalistic sleep in healthy adults. PLoS One. 2018;13(1):e0191883. doi:10.1371/journal.pone.0191883

7. Hoekert M, der Lek RF, Swaab DF, Kaufer D, Van Someren EJ. Comparison between informant-observed and actigraphic assessments of sleep-wake rhythm disturbances in demented residents of homes for the elderly. Am J Geriatr Psychiatry. 2006;14(2):104-111. doi:10.1097/01.JGP.0000192481.27931.c5

8. Tsuchiyama K, Nagayama H, Kudo K, Kojima K, Yamada K Discrepancy between subjective and objective sleep in patients with depression. Psychiatry Clin Neurosci. 2003;57(3):259-264. doi:10.1046/j.1440-1819.2003.01114.x

9. Dayyat EA, Spruyt K, Molfese DL, Gozal D. Sleep estimates in children: parental versus actigraphic assessments. Nat Sci Sleep. 2011;3:115-123. doi:10.2147/NSS.S25676

10. Werner H, Molinari L, Guyer C, Jenni OG. Agreement rates between actigraphy, diary, and questionnaire for children's sleep patterns. Arch Pediatr Adolesc Med. 2008;162(4):350-358. doi:10.1001/ archpedi.162.4.350

11. Poppel KV, Fulton SP, McGregor A, Ellis M, Patters A, Wheless J. Prospective study of the emfit movement monitor. J Child Neurol. 2013;28(11):1434-1436. doi:10.1177/0883073812471858

12. Tenhunen M, Elomaa E, Sistonen H, Rauhala E, Himanen SL. Emfit movement sensor in evaluating nocturnal breathing. Respir Physiol Neurobiol. 2013;187(2):183-189. doi:10.1016/j.resp.2013.03.009 
13. Paajanen M, Välimäki H, Lekkala J. Modelling the electromechanical film (EMFi). J Electrost. 2000;48(3):193-204. doi:10.1016/S03043886(99)00065-0

14. Tenhunen M, Rauhala E, Virkkala J, Polo O, Saastamoinen A, Himanen SL. Increased respiratory effort during sleep is non-invasively detected with movement sensor. Sleep Breath. 2011;15(4):737-746. doi:10.1007/s11325-010-0430-8

15. Alametsä J, Rauhala E, Huupponen E, et al. Automatic detection of spiking events in EMFi sheet during sleep. Med Eng Phys. 2006;28 (3):267-275. doi:10.1016/j.medengphy.2005.07.008

16. Chow CM, Wong SN, Shin M, et al. Defining the rest interval associated with the main sleep period in actigraph scoring. Nat Sci Sleep. 2016;8:321-328. doi:10.2147/NSS.S114969

17. Respironics. Practice aspects of sleep diagnostic. In: Sleep Technician Guide. 2009.

18. Bland JM, Altman DG. Statistical methods for assessing agreement between two methods of clinical measurement. Lancet. 1986;1 (8476):307-310. doi:10.1016/S0140-6736(86)90837-8

19. Haghayegh S, Kang HA, Khoshnevis S, Smolensky MH, Diller KR. A comprehensive guideline for Bland-Altman and intra class correlation calculations to properly compare two methods of measurement and interpret findings. Physiol Meas. 2020;41(5):055012. doi:10.1088/1361-6579/ab86d6

20. de Vet HC, Terwee CB, Ostelo RW, Beckerman H, Knol DL, Bouter LM. Minimal changes in health status questionnaires: distinction between minimally detectable change and minimally important change. Health Qual Life Outcomes. 2006;4(1):54. doi:10.1186/1477-7525-4-54

21. McGraw KO, Wong SP. Forming inferences about some intraclass correlation coefficients. Psychol Methods. 1996;1(1):30-46. doi:10.1037/1082-989X.1.1.30

22. Koo TK, Li MY. A guideline of selecting and reporting intraclass correlation coefficients for reliability research. J Chiropr Med. 2016;15(2):155-163. doi:10.1016/j.jcm.2016.02.012

23. Pollak CP, Tryon WW, Nagaraja H, Dzwonczyk R. How accurately does wrist actigraphy identify the states of sleep and wakefulness? Sleep. 2001;24(8):957-965. doi:10.1093/sleep/24.8.957
24. Sivertsen B, Omvik S, Havik OE, et al. A comparison of actigraphy and polysomnography in older adults treated for chronic primary insomnia. Sleep. 2006;29(10):1353-1358. doi:10.1093/sleep/ 29.10.1353

25. Landis JR, Koch GG. The measurement of observer agreement for categorical data. Biometrics. 1977;33(1):159-174. doi:10.2307/2529310

26. Barouni A, Ottenbacher J, Schneider J, et al. Ambulatory sleep scoring using accelerometers-distinguishing between nonwear and sleep/wake states. PeerJ. 2020;8:e8284. doi:10.7717/peerj.8284

27. Webster JB, Kripke DF, Messin S, Mullaney DJ, Wyborney G. An activity-based sleep monitor system for ambulatory use. Sleep. 1982;5(4):389-399. doi:10.1093/sleep/5.4.389

28. Sadeh A, Lavie P, Scher A, Tirosh E, Epstein R. Actigraphic home-monitoring sleep-disturbed and control infants and young children: a new method for pediatric assessment of sleep-wake patterns. Pediatrics. 1991;87(4):494-499.

29. Sadeh A, Sharkey KM, Carskadon MA. Activity-based sleep-wake identification: an empirical test of methodological issues. Sleep. 1994;17(3):201-207. doi:10.1093/sleep/17.3.201

30. Thoman EB. Sleeping and waking states in infants: a functional perspective. Neurosci Biobehav Rev. 1990;14(1):93-107. doi:10.1016/S0149-7634(05)80165-4

31. Hauri PJ, Wisbey J. Wrist actigraphy in insomnia. Sleep. 1992;15 (4):293-301. doi:10.1093/sleep/15.4.293

32. Fetveit A, Bjorvatn B. Sleep disturbances among nursing home residents. Int J Geriatr Psychiatry. 2002;17(7):604-609. doi:10.1002/gps.639

33. Van de Water AT, Holmes A, Hurley DA. Objective measurements of sleep for non-laboratory settings as alternatives to polysomnography--a systematic review. J Sleep Res. 2011;20(1 Pt 2):183-200. doi:10.1111/j.1365-2869.2009.00814.x

34. Choi BH, Seo JW, Choi JM, et al. Non-constraining sleep/wake monitoring system using bed actigraphy. Med Biol Eng Comput. 2007;45(1):107-114. doi:10.1007/s11517-006-0134-1

35. Sadeh A, Hauri PJ, Kripke DF, Lavie P. The role of actigraphy in the evaluation of sleep disorders. Sleep. 1995;18(4):288-302. doi:10.1093/sleep/18.4.288
Nature and Science of Sleep

\section{Publish your work in this journal}

Nature and Science of Sleep is an international, peer-reviewed, open access journal covering all aspects of sleep science and sleep medicine, including the neurophysiology and functions of sleep, the genetics of sleep, sleep and society, biological rhythms, dreaming, sleep disorders and therapy, and strategies to optimize healthy sleep.
The manuscript management system is completely online and includes a very quick and fair peer-review system, which is all easy to use. Visit http://www.dovepress.com/testimonials.php to read real quotes from published authors. 